\title{
ESTUDIO DE LA EFICACIA DE LATOMA DE MEDIDAS DE VIBRACIONES MECÁNICAS SOBRE SUPERFICIES IRREGULARES Y NO RÍGIDAS
}

\section{STUDY OF THE EFFECTIVENESS OF TAKING MEASUREMENTS OF MECHANICAL VIBRATIONS ON IRREGULAR AND NON-RIGID SURFACES}

Hernández Dávila Eduardo
Escuela Superior Politécnica de Chimborazo. edhernandez@espoch.edu.ec https://orcid.org/0000-0003-4899-2371
Olivo Malliquinga Danilo
Escuela Superior Politécnica de Chimborazo. https://orcid.org/0000-0001-7577-7584
Carrillo Villa Cristian

Escuela Superior Politécnica de Chimborazo. https://orcid.org/0000-0003-3184-337X

\section{RESUMEN}

El objetivo de la presente investigación experimental fue evaluar el valor eficaz de las mediciones de velocidad global de vibración obtenidas en cuatro puntos diferentes de superficie irregular y no rígida, en comparación con las obtenidas sobre un inserto plano y rígido utilizado como referencia. Todas las mediciones se efectuaron en el plano radial vertical del rodamiento del lado libre del motor eléctrico de un módulo de pruebas funcionando bajo condiciones de trabajo constantes. La normalidad de los datos obtenidos fue evaluada mediante la prueba de Shapiro Wilk, y la comparación de las muestras se efectuó aplicando el estadístico de prueba de t student. Como resultado se obtuvo que las mediciones sobre superficies irregulares y no rígidas son diferentes a las obtenidas sobre insertos, aportando con evidencia concluyente que respalda el cuidado que debe existir al momento de acoplar un sensor de análisis vibracional sobre la superficie de la máquina a inspeccionar.

Palabras clave: medición de vibración, análisis vibracional, valor eficaz, inserto.

\section{ABSTRACT}

The objective of this experimental investigation was evaluate the effective value of the global vibration velocity measurements obtained at four different points of irregular and non-rigid surface, compared to those obtained on a flat and rigid insert used as a reference. All measurements were made in the vertical radial plane of the bearing on the free side of the electric motor of a test module operating under constant working conditions. The normality of the data obtained was evaluated by the Shapiro Wilk test, and the comparison of the samples was carried out by applying the $t$ student test statistic, where it was obtained as a result that the measurements on irregular and non-rigid surfaces are different to those obtained on inserts, providing conclusive evidence that supports the care that must exist when attaching a vibrational analysis sensor on the surface of the machine to be inspected.

Keywords: vibration measurement, vibrational analysis, effective value, insert 


\section{INTRODUCCIÓN}

En el diagnóstico técnico de máquinas rotacionales es común la aplicación de análisis de vibraciones para diagnosticar las fallas, en el que se emplean distintos tipos de sensores, el más utilizado es el acelerómetro con de bases magnéticas cuya correcta utilización se obtiene colocando este sensor sobre puntos rígidos y lisos. En el caso que la máquina a analizar carezca de estos puntos se instalan insertos o targets (Vassileva, 2007).

La vibración es el movimiento armónico de una máquina, o elemento de ella, en cualquier dirección del espacio desde su posición de equilibrio (Royo et al., 2000). Los parámetros característicos de las vibraciones son el desplazamiento que indica la cantidad de movimiento que la masa experimenta con respecto a su posición de reposo, el periodo es el tiempo que tarda la masa en realizar un ciclo completo, la frecuencia es el número de ciclos que ocurren en una unidad de tiempo, velocidad que se refiere a la proporción del cambio de posición con respecto al tiempo y la aceleración proporciona la medida del cambio de la velocidad con respecto al tiempo (Royo et al, 2000; AENOR, 2009; Olarte et al., 2010).

Los datos que se deben considerar, desplazamiento, velocidad o aceleración dependen de la velocidad de la máquina, de acuerdo con su relación equivalente de frecuencia (rpm=cpm), así, para bajas rpm, se toman datos de desplazamientos (Ebersbach, 2008). Para valores que estén dentro del orden de 600 y 60.000 rpm, se miden velocidades, y los de orden superior, se consideran las aceleraciones (Moreno et al., 2014; Olarte et al., 2010).

Existen motores de molinos que trabajan bajo niveles de vibración aceptables que generalmente no superan los $2 \mathrm{~mm} / \mathrm{s}$, por esta razón se deben tener en cuenta en las mediciones que superan estos valores, aunque están dentro de lo normal, por lo que es necesario definir el contexto operacional de la máquina donde se especifican los parámetros normales de operación (Torres y Batista, 2010; Martin et al., 2018).

También se aplica la medición de vibraciones para realizar diagnóstico técnico de problemas más serios en las máquinas y estructuras, donde el riesgo de una falla puede ser catastrófico debido a la generación de grietas con antecedentes en la literatura del colapso de plantas debido a ejes y estructuras agrietadas (Zepeng, 2020).

Con el análisis de vibración se puede determinar problemas específicos de los rodamientos como grietas en la pista interna, en la pista externa, problemas de rodadura y canasta que presentan armónicos específicos generados por grietas en la estructura (Zepeng, 2020). Al momento de realizar el análisis de vibraciones en máquinas, se debe conocer datos técnicos, historial de mantenimiento, criticidad, debido que es una metodología con un alto valor de diagnóstico. (Iriondo, 2007; González, 2019).

En la bibliografía consultada se pone énfasis en las aplicaciones del análisis de vibracional, sin embargo, no se resalta la importancia que tiene la repetitividad de las mediciones la cual se define como la proximidad de concordancia entre los resultados de mediciones sucesivas del mismo mensurado bajo las mismas condiciones de mediciones y para este requerimiento es necesario colocar el sensor de manera adecuada (Portuondo y Portuondo, 2010; Saavedra, 2011; García, 2014).

En este sentido, el objetivo de la presente investigación fue evaluar la repetitividad de las mediciones del módulo de pruebas colocando el sensor en puntos cercanos al rodamiento y sobre un inserto. Los datos de las muestras obtenidas se compararon con el estadístico paramétricos t-student.

\section{MATERIALES Y MÉTODOS}

Para investigar los errores en la toma de medidas de vibraciones de motores eléctricos mediante acelerómetros y bases magnéticas, se usarón como referencias las medidas que se obtienen en los insertos de acuerdo con las sugerencias que se establecen en normas internacionales que recomiendan colocar los sensores lo más cercano a los rodamientos en una base rígida y lisa asegurando su montaje completo para obtener medidas exactas (Estupiña et al., 2006; Peral Orts et al., 2008).

La medición de referencia está constituida por la utilización de un inserto circular de acero ANSI 1020 de 3 mm de espesor, diámetro de 25 $\mathrm{mm}$ y con superficie lisa en la cual se asentó el sensor. 
Al desarrollar el experimento se utilizó el módulo de prueba de la figura 1, que consiste en un motor trifásico jaula de ardilla con potencia de $373 \mathrm{~W}$ de cuatro polos magnéticos anclado sobre una base de acero ANSI 1020 de $10 \mathrm{~mm}$ de espesor. Para la alimentación se empleó tablero eléctrico que controla directamente el encendido y apagado del motor (Almeida, 2010).

El sensor utilizado consta internamente de un material piezo eléctrico que genera electricidad proporcional a la presión aplicada sobre el mismo por las vibraciones del motor, además cuenta con un electro imán de 16 libras que asegura el sensor al inserto. El sensor y analizador de vibraciones VIBRACHECK se encargó de recolectar los datos de vibraciones en los puntos antes mencionados (IDEAR, 2019). El análisis de datos se realizó con el software del MAINTraq Predictive que incluye el analizador de vibraciones (EVTECH, 2020; Pernía, 2004).

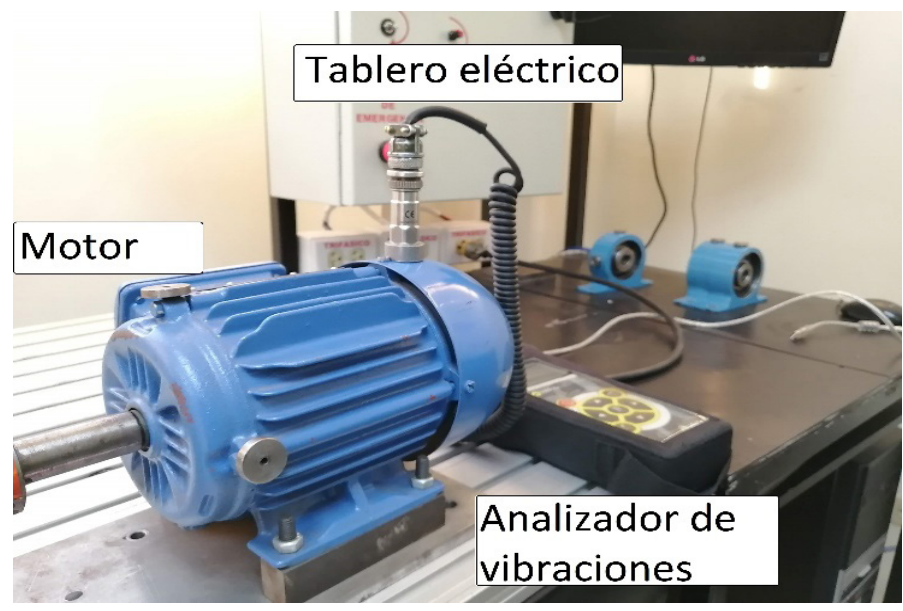

Figura 1. Módulo de pruebas

En la figura 2 se especifican los puntos en los cuales se realizaron las mediciones que se usaron:

a. Punto 1, medida de referencia con sensor montado en el inserto.

b. Punto 2, sobre el punto 1 pero quitando el inserto.

c. Punto 3, sobre la tapa del motor.

d. Punto 4, sobre las aletas del motor.

e. Punto 5, sobre la superficie curva de la carcasa del motor.

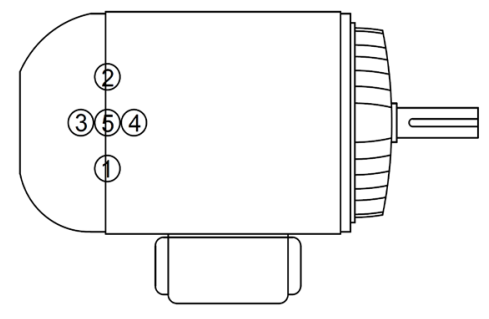

Figura 2. Esquema de los puntos de medición

Para el análisis estadístico se utilizó el software $\mathrm{R}$, donde se evaluó la normalidad de los datos y se compararon las muestras (Hernández Dávila et al., 2019).

\section{RESULTADOS}

El valor eficaz de las cincuenta medidas de velocidad global de vibraciones de los distintos puntos del plano radial vertical del rodamiento del lado libre del motor eléctrico y del módulo de pruebas funcionando bajo condiciones de trabajo constantes, se pueden apreciar en la tabla 1.

Tabla 1. Mediciones de la velocidad global de vibración en los diferentes puntos

\begin{tabular}{|c|c|c|c|c|c|}
\hline $\mathbf{N}^{\circ}$ & $\begin{array}{c}\mathbf{I n s e r t o} \\
{[\mathbf{m m} / \mathbf{s}]}\end{array}$ & $\begin{array}{c}\text { Sin } \\
\text { inserto } \\
{[\mathbf{m m} / \mathbf{s}]}\end{array}$ & $\begin{array}{c}\text { Tapas } \\
{[\mathbf{m m} / \mathbf{s}]}\end{array}$ & $\begin{array}{c}\text { Aletas } \\
{[\mathbf{m m} / \mathbf{s}]}\end{array}$ & $\begin{array}{c}\text { Superficie } \\
\mathbf{C u r v a} \\
{[\mathbf{m m} / \mathbf{s}]}\end{array}$ \\
\hline 1 & 8,502 & 8,171 & 10,728 & 4,082 & 4,543 \\
\hline 2 & 8,595 & 8,289 & 10,759 & 4,058 & 5,157 \\
\hline 3 & 8,493 & 8,226 & 10,593 & 4,074 & 4,879 \\
\hline 4 & 8,547 & 8,252 & 10,659 & 4,152 & 4,966 \\
\hline 5 & 8,564 & 8,188 & 10,888 & 4,199 & 5,434 \\
\hline 6 & 8,529 & 8,263 & 10,803 & 4,037 & 5,059 \\
\hline 7 & 8,637 & 8,246 & 10,594 & 4,145 & 4,515 \\
\hline 8 & 8,519 & 8,161 & 10,630 & 4,166 & 4,450 \\
\hline 9 & 8,546 & 8,187 & 10,781 & 4,131 & 4,689 \\
\hline 10 & 8,447 & 8,259 & 10,688 & 4,067 & 4,523 \\
\hline$\vdots$ & $\vdots$ & $\vdots$ & $\vdots$ & $\vdots$ & $\vdots$ \\
\hline 50 & 8,215 & 8,173 & 4,217 & 8,565 & 10,584 \\
\hline
\end{tabular}


FIGEMPA: Investigación y Desarrollo / Año 2020 / Volumen 1 / Número 1 / junio 2020 / pág. 93-98/ Hernández E., Olivo D., Carrillo C.

Los resultados del análisis estadístico de los datos indicados en la tabla 1 , se observan en la tabla 2 , cuyos respectivos histogramas de frecuencias, están ilustrados en la figura 3.

Tabla 2. Valores estadísticos de las mediciones obtenidas

\begin{tabular}{|l|c|c|c|c|}
\hline $\begin{array}{c}\text { UBICACIÓN } \\
\text { DE } \\
\text { SENSOR }\end{array}$ & $\begin{array}{c}\text { MÍNIMO } \\
{[\mathrm{mm} / \mathrm{s}]}\end{array}$ & $\begin{array}{c}\text { MÁXIMO } \\
{[\mathrm{mm} / \mathrm{s}]}\end{array}$ & $\begin{array}{c}\text { MEDIA } \\
{[\mathrm{mm} / \mathrm{s}]}\end{array}$ & $\begin{array}{c}\text { DESVIACIÓN } \\
\text { ESTÁNDAR }\end{array}$ \\
\hline Inserto & 8,373 & 8,661 & 8,518 & 0,0652804 \\
\hline Sin inserto & 8,085 & 8,289 & 8,186 & 0,0418837 \\
\hline Tapa & 10,25 & 11,07 & 10,71 & 0,1832880 \\
\hline Aletas & 3,926 & 4,217 & 4,074 & 0,0739275 \\
\hline $\begin{array}{l}\text { Superficie } \\
\text { Curva }\end{array}$ & 3,031 & 7,021 & 4,929 & 0,7638764 \\
\hline
\end{tabular}

Para poder seleccionar el método para la comparación de las muestras es imprescindible que primeramente se realice una prueba de normalidad de los datos, para tal propósito, se decidió utilizar el método de Shapiro Wilk (Sánchez, 2015; Saldaña, 2016), por su aceptable efectividad y amplia utilización. Estos resultados se indican en la tabla 3 y se visualiza en la figura 4.

Tabla 3. Resultado prueba de normalidad

\begin{tabular}{|l|c|c|l|}
\hline $\begin{array}{l}\text { UBICACIÓN } \\
\text { DE SENSOR }\end{array}$ & W & P-VALOR & NORMALIDAD \\
\hline Inserto & 0,98012 & $0,5572>0,05$ & $\begin{array}{l}\text { Si se distribuye } \\
\text { normalmente }\end{array}$ \\
\hline Sin inserto & 0,98088 & $0,5515>0,05$ & $\begin{array}{l}\text { Si se distribuye } \\
\text { normalmente }\end{array}$ \\
\hline Tapa & 0,93194 & $0,9234>0,05$ & $\begin{array}{l}\text { Si se distribuye } \\
\text { normalmente }\end{array}$ \\
\hline Aletas & 0,98043 & $0,5701>0,05$ & $\begin{array}{l}\text { Si se distribuye } \\
\text { normalmente }\end{array}$ \\
\hline $\begin{array}{l}\text { Superficie } \\
\text { curva }\end{array}$ & 0,93824 & $0,1590>0,05$ & $\begin{array}{l}\text { Si se distribuye } \\
\text { normalmente }\end{array}$ \\
\hline
\end{tabular}
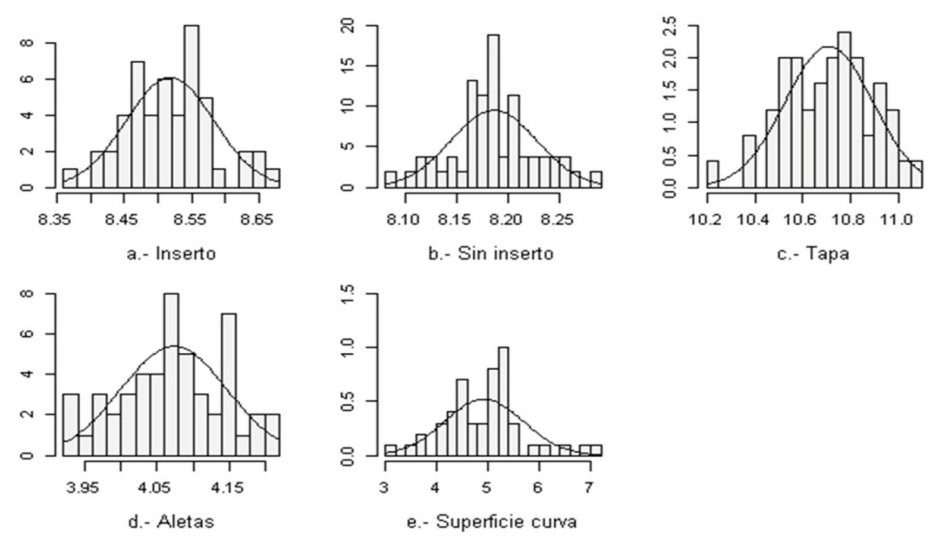

Figura 5. Histogramas de las pruebas de normalidad
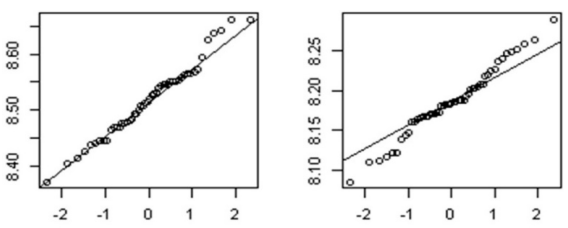

b.- Sin inserto
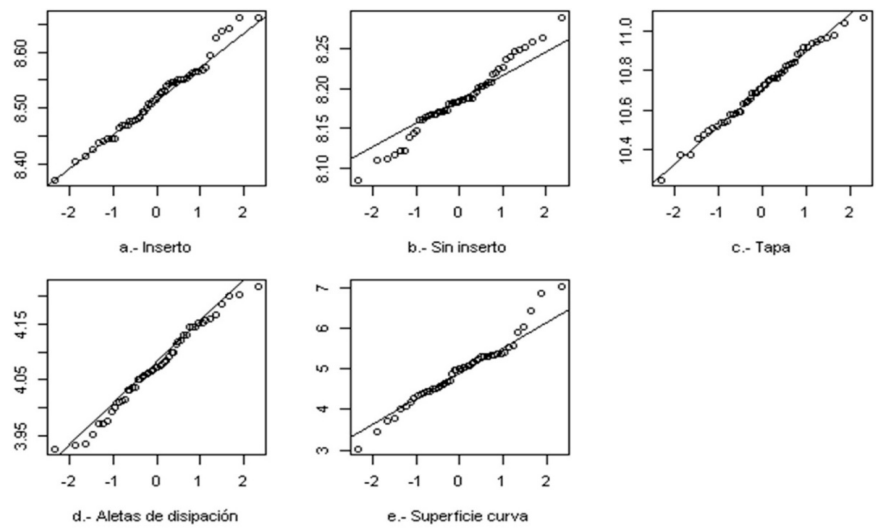

- Tapa

Figura 4. Gráfico cuantil - cuantil de las muestras

Dado que en las pruebas de Shapiro-Wilk se encontró que todos los datos se distribuyen normalmente, se aplicó, el método paramétrico de comparación de muestras $t$ student (Sánchez Turcios 2015), de manera de realizar la demostración de la hipótesis; en donde la Ho corresponde a la igualdad de las muestras, que indica que la diferencia en la ubicación del acelerómetro no afecta a la eficacia de las medidas tomadas y cuyos resultados se muestran en la tabla 4 .

\section{Tabla 4. Comparación de variables}

\begin{tabular}{|l|c|c|c|}
\hline $\begin{array}{l}\text { VARIABLES } \\
\text { COMPARADAS }\end{array}$ & $\mathbf{T}$ & DF & P-VALOR \\
\hline $\begin{array}{l}\text { Inserto; Sin } \\
\text { inserto }\end{array}$ & 30,528 & 82,696 & $2,2 \mathrm{e}-16<0,01$ \\
\hline Inserto; Tapa & $-79,641$ & 61,235 & $2,2 \mathrm{e}-16<0,01$ \\
\hline Inserto; Aletas & 318,64 & 96,522 & $2,2 \mathrm{e}-16<0,01$ \\
\hline $\begin{array}{l}\text { Inserto; } \\
\text { Superficie curva }\end{array}$ & 33,104 & 49,716 & $2,2 \mathrm{e}-16<0,01$ \\
\hline
\end{tabular}

Figura 5. Comparación de las muestras mediante el diagrama de cajas 


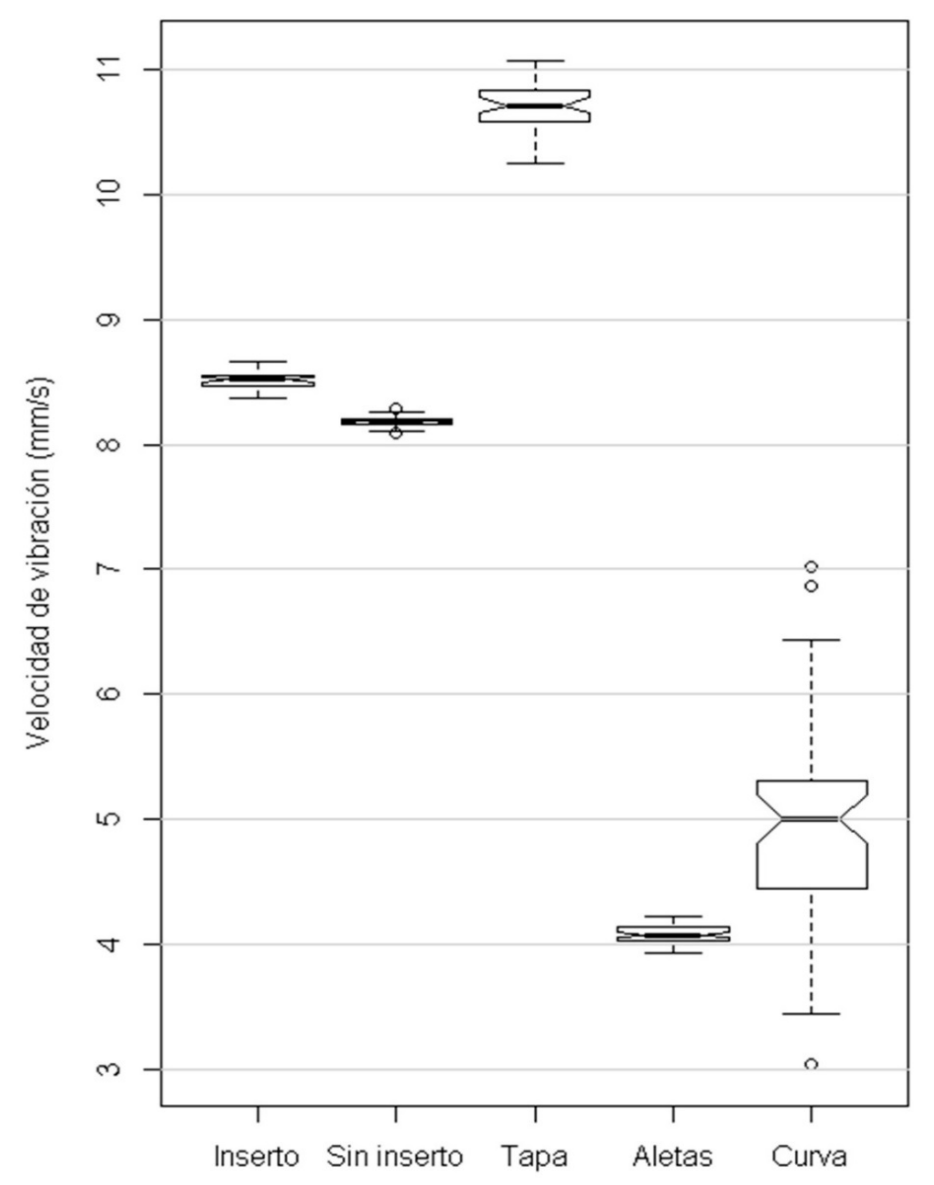

Ubicación del acelerómetro

\section{DISCUSIÓN}

La tabla 2 revela que las medidas de velocidad de vibración obtenidas sobre el punto sin inserto son las menos dispersas puesto que su desviación estándar es 0,0418837 , que es menor a los otros grupos de datos y su media se distancia muy poco de la media de las medidas obtenidas sobre el inserto. Por otro lado, las medidas más dispersas corresponden a las realizadas sobre la superficie curva, por tanto, son las menos confiables desde el punto de vista de la repetitividad.

En conformidad con los resultados expuestos en la tabla 3 y figura 4 , las cincuenta mediciones que conforman las muestras de la velocidad global de vibración de los distintos puntos donde se colocó el sensor, se distribuyen normalmente con el 95\% de confianza, por lo que se justifica la utilización del método paramétrico t student para la demostración de la hipótesis.

En la evaluación de hipótesis, el p-valor obtenido con la prueba $t$ student mediante el software estadístico $R$, es menor que 0,01 ; por lo tanto, se rechaza la Ho y se concluye que las muestras son diferentes con el $99 \%$ de confianza; por consecuencia, la diferencia en la ubicación del acelerómetro afecta significativamente a la eficacia de las mediciones.

\section{CONCLUSIONES}

Las mediciones de la velocidad global de vibración extraídas sobre superficies irregulares y no rígidas arrojan valores diferentes a las que se obtienen mediante la utilización de insertos, por lo que se concluye que las mediciones sobre estas superficies irregulares y no rígidas son ineficaces.

Las superficies irregulares y no rígidas generan un área de contacto incompleta e inestable con la base magnética del sensor; por lo que se deduce que se llega al mismo problema con cualquier factor que cause mal contacto como por ejemplo presencia de suciedad o lubricantes en la superficie del punto de sujeción del sensor.

La evidencia aportada en la presente investigación ratifica el cuidado que debe existir al momento de acoplar un sensor de análisis vibracional sobre la superficie de la máquina a inspeccionar.

\section{REFERENCIAS BIBLIOGRÁFICAS}

AENOR. (2009). ISO 10816-3:2009. Evaluation of machine vibration. AENOR.

Almeida, C. (2010). Diseño y construcción de un banco de pruebas para diagnosticar fallas en engranajes mediante espectros vibracionales para el laboratorio de mantenimiento de la Facultad de Mecánica. Escuela Superior Politécnica de Chimborazo. Riobamba. Ecuador.

Ebersbach S. (2008). Desarrollo de sistemas expertos para el análisis de vibraciones en el monitoreo del estado de la máquina. Sistemas Expertos con Aplicaciones. Vol. 34(1): 291-299.

Estupiñan, E., San Martin, C. y Solaligue, R. (2006). Diseño e implementación de un analizador virtual. 
Revista de la Facultad de Ingeniería on line. Vol. 14(1): 7-15.

EVTECH. (2020). Principios de funcionamiento de sensores de proximidad en maquinaria rotativa [Online]. [cited 2020 01/15]. Disponible en: https://evtech.cl/ principio-de-funcionamiento-de-sensores-de-proximidad-en-maquinaria-rotativa.

García MJG. (2014). Metodología de selección de indicadores. Tesis doctoral. Escuela Politécnica Superior Universidad Carlos III de Madrid, Departamento de Ingeniería Mecánica. Madrid. España.

González, R. (2019). Incremento en disponibilidad y control de almacén mediante un análisis del historial del mantenimeinto. Revista de Operaciones Tecnológicas. Vol. 3(10): 14-18.

Hernández Dávila, E.; Cajamarca-Villa, J.; CacuangoEugenio, L.; López - Pérez, V. (2019). Estimación empírica del pretensado de una banda trapezoidal mediante el deslizamiento de las poleas. INGENIUS Revista de Ciencia y Tecnología. Vol. 21: 63-70.

IDEAR. (2019). VIBRA chek 200. Analizador de vibraciones mecánicas de dos canales. Manual. IDEAR condition monitoring.

Iriondo MR. (2007). Vibraciones mecánicas, qué son y cómo se evalúa el riesgo. DYNA Ingeniería e Industria. Vol. 82: 499-506.

Martín, DI.; Nadra, A.; Senar, P. (2018). Las nuevas realidades del diseñador en el contexto de la digitalizacion industrial. Actas de Jornadas de Investigacion. Tecnologia en relacion proyectual. Buenos Aires. Argentina. Disponible en https://publicacionescientificas.fadu.uba.ar/ index.php/actas/article/view/563/813. Fecha de acceso 16-05-2020.

Moreno García, F.; Becerra-Vargas, J.; Rendón-Echeverri, C.; Moreno, F. (2014). Diseño de un sistema de análisis temporal y espectral para detectar fallas por vibración en motores eléctricos. Revista Facultad de Ingeniería 24(38):41.

Olarte, W.; Botero, A.; Cañón, M.; Benhur, A (2010). Análisis de vibraciones: Una herramienta clave en el mantenimiento predictivo. Scientia et Technica. Vol. XVI (45): 219-222.

Peral Orts, R.; Campillo Davó, N.; Velasco Sánchez, E. (2008). Bancos de ensayos para docencia en vibraciones mecánicas. Acústica. 2008. Universidad de Coimbra. Coimbra. Portugal.

Pernía A. (2004). Introducción a la medición de vibración. Tesis de maestría. Facultad de Ingeniería. Universidad de los Andes. Mérida. Venezuela.

Portuondo Paisan, Y.; Portuondo, J (2010). La Repetibilidad y Reproducibilidad en el Aseguramiento de la Calidad de los Procesos de Medición. Tecnología Química. Vol. XXX (2): 117-121.

Royo, J.; Rabanaque, G.; Torres, F. (2000). Análisis de vibraciones e interpretación de datos. DIDYF Universidad de Zaragoza. Zaragoza. España.

Saavedra PN. (2011) La medición y análisis de las vibraciones como técnica de inspección de equipos y componentes, aplicaciones, normativas y certificación. [Online]. Disponible en http://www.aaende.org.ar/sitio/material/ CONFCHILE.pdf. Fecha de acceso: 12 de mayo, 2020.

Saldaña R. (2016). Pruebas de bondad de ajuste a una distribución normal. Revista Enfermería del trabajo. Vol. 6(3): 105-114.

Sánchez Turcios, R. (2015). T-Student. Usos y abusos. Revista Mexicana de Cardiología. Vol. XXVI (1): 59-61.

Sánchez, RA. (2015). Prueba de Wilcoxon-Mann-Whitney: mitos y realidades. Revista Mexicana de Endocrinología, Metabolismo \& Nutrición. Vol 2 (1) 18-21.

Torres, RM; Batista, C. (2010). Análisis vibro dinámico de motores eléctricos. Ingeniería Mecánica. Vol. 13(1): 9-8.

Vassileva MP. (2007). Mantenimiento y Análisis de vibraciones. Ciencia y Sociedad. Vol. XXXII (4): 668-678.

Zepeng, L. (2020). Análisis de vibraciones para la detección de fallas en cojinetes de palas de turbinas eólicas a gran escala con un método empírico de umbralización de wavelet. Renewable Energy. Vol. 146: 99-110. 